\title{
BMJ Open Factors associated with attendance at screening for breast cancer: a systematic review and meta-analysis
}

\author{
Rebecca Mottram, ${ }^{1}$ Wendy Lynn Knerr (D) , ${ }^{1}$ Daniel Gallacher (D) , ${ }^{1}$ Hannah Fraser, ${ }^{1}$ \\ Lena Al-Khudairy, ${ }^{1}$ Abimbola Ayorinde (D) , ${ }^{1}$ Sian Williamson, ${ }^{1}$ Chidozie Nduka, ${ }^{1}$ \\ Olalekan A Uthman (D) , ${ }^{1}$ Samantha Johnson, ${ }^{2}$ Alexander Tsertsvadze, ${ }^{3}$ \\ Christopher Stinton, ${ }^{1}$ Sian Taylor-Phillips (D) , ${ }^{1}$ Aileen Clarke ${ }^{1}$
}

To cite: Mottram R, Knerr WL, Gallacher D, et al. Factors associated with attendance at screening for breast cancer: a systematic review and meta-analysis. BMJ Open 2021;11:e046660. doi:10.1136/ bmjopen-2020-046660

- Prepublication history and additional supplemental material for this paper are available online. To view these files, please visit the journal online (http://dx.doi.org/10.1136/ bmjopen-2020-046660)

Received 05 November 2020 Accepted 17 September 2021

A Check for updates

(C) Author(s) (or their employer(s)) 2022. Re-use permitted under CC BY. Published by BMJ.

${ }^{1}$ Division of Health Sciences, Warwick Medical School, University of Warwick, Coventry, UK

${ }^{2}$ University of Warwick Library, University of Warwick, Coventry, UK

${ }^{3}$ School of Epidemiology and Public Health, University of Ottawa Faculty of Medicine, Ottawa, Ontario, Canada

Correspondence to

Aileen Clarke;

aileen.clarke@warwick.ac.uk

\section{ABSTRACT}

Objective Attendance at population-based breast cancer (mammographic) screening varies. This comprehensive systematic review and meta-analysis assesses all identified patient-level factors associated with routine population breast screening attendance.

Design CINAHL, Cochrane Library, Embase, Medline, OVID, PsycINFO and Web of Science were searched for studies of any design, published January 1987-June 2019, and reporting attendance in relation to at least one patient-level factor.

Data synthesis Independent reviewers performed screening, data extraction and quality appraisal. OR and 95\% Cls were calculated for attendance for each factor and random-effects meta-analysis was undertaken where possible.

Results 0 f 19776 studies, 335 were assessed at full text and 66 studies ( $n=22150$ 922) were included. Risk of bias was generally low. In meta-analysis, increased attendance was associated with higher socioeconomic status (SES) ( $n=11$ studies; OR 1.45, 95\% Cl: 1.20 to 1.75); higher income ( $\mathrm{n}=5$ studies; OR 1.96, 95\% Cl: 1.68 to 2.29); home ownership ( $\mathrm{n}=3$ studies; OR $2.16,95 \% \mathrm{Cl}: 2.08$ to 2.23); being non-immigrant ( $\mathrm{n}=7$ studies; OR 2.23, $95 \%$ Cl: 2.00 to 2.48 ); being married/cohabiting ( $n=7$ studies; OR $1.86,95 \% \mathrm{Cl}: 1.58$ to 2.19 ) and medium (vs low) level of education ( $\mathrm{n}=6$ studies; OR 1.24, $95 \% \mathrm{Cl}$ : 1.09 to 1.41 ). Women with previous false-positive results were less likely to reattend ( $\mathrm{n}=6$ studies; OR $0.77,95 \% \mathrm{Cl}: 0.68$ to 0.88 ). There were no differences by age group or by rural versus urban residence.

Conclusions Attendance was lower in women with lower SES, those who were immigrants, non-homeowners and those with previous false-positive results. Variations in service delivery, screening programmes and study populations may influence findings. Our findings are of univariable associations. Underlying causes of lower uptake such as practical, physical, psychological or financial barriers should be investigated.

Trial registration number CRD42016051597.

\section{INTRODUCTION}

Breast cancer was the most commonly diagnosed cancer worldwide in 2020, with 2.3 million cases, and the most common cause of

\section{STRENGTHS AND LIMITATIONS OF THIS STUDY}

$\Rightarrow$ Comprehensive systematic review of all identified patient-level factors associated with attendance at routine population-based breast cancer (mammographic) screening.

$\Rightarrow$ Two reviewers independently conducted all study selection, data extraction and quality appraisal using Quality in Prognosis Studies.

$\Rightarrow$ Both observational and experimental designs were included, using control arms of quasi-experimental or randomised designs and ORs were independently recalculated using each study's raw data.

$\Rightarrow$ Heterogeneity is high partly due to the large size of studies. Studies were separately meta-analysed by study design, and sensitivity analysis was conducted for one study with an extreme effect size.

$\Rightarrow$ Reporting of potential confounders and effect modifiers was highly variable in studies; this was partially mitigated by recategorising variables, such as education levels, to harmonise variables across studies where possible.

cancer death in women. ${ }^{1}$ Breast cancer incidence is higher in more developed countries (Europe, Australia, New Zealand and North America; 55.9 cases per 100000 population) than in less developed countries (29.7 per $100000)$, while the reverse is true of death rates (12.4 vs 15.0 per 100000 , respectively). ${ }^{1}$ In the EU, mortality rates decreased $18.7 \%$ between the period 2005-2009 and 2019 from 16.44 to (predicted) 13.36 per $100000 .^{2}$

Population-based mammographic screening aims to reduce breast cancer mortality. However, there has been controversy about the balance of benefits and harms of breast screening ${ }^{3}$ and breast screening programmes have become more aware of the need for promoting informed choice. ${ }^{45}$

Attendance at breast screening is not uniform among the eligible population. ${ }^{6}$ Ross $e t a l^{7}$ described attendance at screening 
as an individual decision (behavioural) which is affected by accessibility of services (structural) and by a woman's immediate surroundings (societal). Characteristics that have been associated with screening attendance can be grouped into a number of categories related to sociodemographic factors; health status; health behaviours; accessibility and logistics; beliefs, attitudes and knowledge; simple intention to attend and societal factors including health systems financing and organisation. ${ }^{8-11}$

Most reviews of factors associated with breast screening attendance have focused on individual factors. ${ }^{12-14}$ We aimed to provide a comprehensive systematic review of all identified patient-level characteristics associated with the uptake of population-based mammographic screening, to inform screening programmes of the available evidence about who does and does not attend.

\section{METHODS}

\section{Protocol and registration}

The review was conducted in accordance with prespecified methods documented in the protocol registered on the 22November 2016 in the PROSPERO International Prospective Register of Systematic Reviews database (online supplemental file A). ${ }^{15}$

\section{Search and information sources}

The Cumulative Index to Nursing and Allied Health Literature (CINAHL), Cochrane Library, Embase, Medline, PsycINFO and Web of Science were searched for studies published between 1 January 1987 and 26 June 2019. The search was developed in Medline using a combination of MeSH headings and free-text terms and adapted for use in the other databases (the search strategy is available in online supplemental file B).

Reference lists of relevant reviews were searched for potentially relevant studies. Experienced researchers with prior studies in the field were contacted to identify other potentially relevant studies that had not been identified in the searches.

\section{Eligibility criteria}

Primary studies of any design were included if they reported attendance data from routine populationbased mammography screening programmes in relation to at least one patient-level factor, and were written in English between January 1987 and June 2019. Studies were excluded if they involved self-reported mammography uptake, opportunistic screening programmes, data for only a subgroup of the eligible population (eg, only women in a narrow age range, only immigrants or only rural women) or uptake data by number of invitations sent rather than number of women. Reviews, commentaries, opinions, letters, and non-empirical and qualitative studies were excluded.

\section{Study selection and data extraction process}

Pairs of reviewers screened titles and abstracts independently to identify potentially relevant studies with third reviewer cross-check. Two reviewers independently assessed full-text studies for formal inclusion/exclusion assessment against predefined eligibility criteria with third reviewer cross-check. Disagreements were resolved by a consensus between the two reviewers or by help of a third reviewer.

Data from included studies were extracted and then cross-checked by two reviewers independently. The data included the number of women who attended mammographic screening and the number invited, and data on patient characteristics, including: sociodemographic factors, such as age, marital status, educational level, race/ethnicity, immigration status and socioeconomic status (SES, which was measured in two ways, (a) with various composite indices of deprivation that included factors such as housing density, employment, education, social support, car ownership and crime prevalence, and (b) based on household income); beliefs, attitudes and socioemotional factors; health history and behaviours; logistic and accessibility factors (eg, distance from screening centre).

\section{Risk of bias of included studies}

Risk of bias (RoB) of all included studies was appraised by two independent reviewers using the Quality in Prognosis Studies (QUIPS) tool. ${ }^{16}$ The QUIPS tool covers six RoB domains (participation, attrition, prognostic factor, confounding factors, outcome measurement and analysis and reporting), each of which includes multiple items that are judged separately. A conclusive judgement for each RoB domain is reached and expressed on a threegrade scale (high, moderate or low RoB).

\section{Synthesis of data}

We used raw attendance data to calculate unadjusted ORs for each factor. A random-effects model-based meta-analysis was conducted for an association between a factor of interest (dichotomous or more categories) and the dichotomous outcome (screening attendance) to generate Mantel-Haenszel ORs with $95 \%$ CIs, when possible. ${ }^{17}$ Random-effects models were used to allow for heterogeneity in the effects of the factors considered to vary across the different studies.

In addition to the main meta-analyses, we conducted separate meta-analyses for (a) observational studies whose samples were made up only of women who had previously attended screening (hereafter referred to as rescreening studies) and (b) intervention studies (quasiexperimental and randomised controlled trials) that reported characteristics separately for intervention and control arms, recording only data for the control group, as their attendance would not be influenced by exposure to an intervention. We also conducted a sensitivity analysis to determine the impact of a study with an extreme effect size ${ }^{18}$ on the meta-analysis of SES.

We summarised results narratively if there were inadequate quantitative data for meta-analysis, if variables were reported in fewer than three studies, ${ }^{17}$ or if the data from 


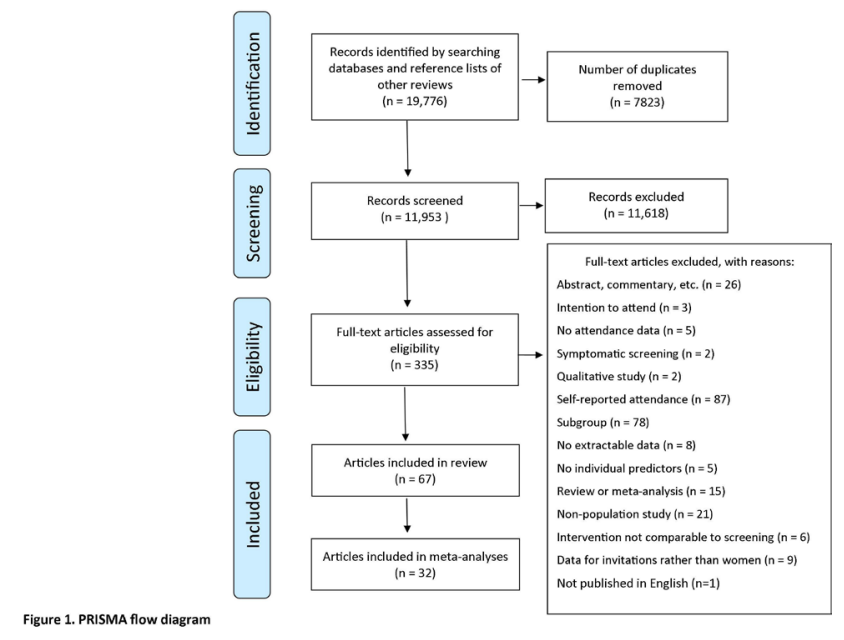

Figure 1 PRISMA flow diagram, showing the process of study flow and reasons for exclusion. The searches of electronic databases identified 11953 unique publications (after deduplication), published between January 1987 and June 2019, of which 11618 were excluded at the level of abstract/title screening, leaving 335 records for full-text review. Of the 335 full texts, 66 unique studies reported in 67 publications were included. PRISMA, Preferred Reporting Items for Systematic Reviews and Meta-Analyses.

multiple studies were highly variable and therefore could not be meaningfully pooled.

This review is reported according to Preferred Reporting Items for Systematic Reviews and Meta-Analyses guidelines (online supplemental file C). ${ }^{19}$ All analyses were conducted in Stata V.16.

\section{Patient and public involvement}

Public contributors were involved in design and informed of ongoing progress and findings as part of the West Midlands Centres for Leadership in Applied Health Research. Results were reported back to the contributors as part of the wider dissemination activities of the relevant theme in the Centres for Leadership in Applied Health Research.

\section{RESULTS}

\section{Literature search}

The process of study flow and reasons for exclusion are provided in figure 1. In brief, the searches of electronic databases identified 11953 unique publications (after deduplication), published between January 1987 and June 2019, of which 11618 were excluded at the level of abstract/title screening, leaving 335 records for full-text review. Of the 335 full texts, 66 unique studies reported in 67 publications were included. ${ }^{180-87}$

\section{Study characteristics}

Characteristics of all included studies are listed in online supplemental file D. Of the 66 studies, 49 were observational (45 retrospective cohort, 2 cross-sectional and 2 case-control designs); and 17 were intervention studies (16 randomised controlled trials and 1 quasiexperimental). Sample sizes ranged from 82 to 4.8 million.

The studies were conducted in Europe $(n=40)$, North America $(n=18)$, Asia-Pacific $(n=5)$ and the Middle East $(n=3)$. The UK had the most studies $(n=16)$ followed by the USA $(n=11)$.

We were able to pool data from 31 observational studies (reported in 32 publications) on the attendance at screening in relation to nine factors (age, education, home ownership, immigration status, marital status, results of previous mammogram, rural/urban residence, SES and income) (table 1). We were only able to pool data from three intervention studies, and only for one factor (age).

Adequate data for meta-analysis was not provided for 35 studies; although six of these studies provided adequate data to calculate ORs and CIs, and are narratively reported in table 2. The remaining 29 studies reported data that could not be analysed. (Reasons are detailed in online supplemental file E.) In brief, 14 of the 29 studies were intervention trials, where data were not in the right format for us to use. The other 15 studies could not be analysed because uptake data were reported by healthprovider characteristics rather than patient characteristics; because the paper reported percentage uptake but not sample sizes per category; or because data for different factors were not reported separately.

\section{Risk of bias}

RoB across studies was generally low on all domains (figure 2). For study participation, $71 \%$ of studies were considered at low RoB; for attrition, $91 \%$; for outcome measurement, $97 \%$ and for statistical analysis and reporting, $83 \%$. For measurement of variables associated with attendance (prognostic factors), more than half $(61 \%)$ of studies had a low RoB, while $23 \%$ had a high RoB, mostly due to SES being measured at the area level (eg, neighbourhood) rather than at the individual level. More than half of studies (53\%) had a low RoB with regard to measuring potential confounders, with around one-quarter $(27 \%)$ having a moderate risk and just over one-fifth $(21 \%)$ having a high risk.

\section{Quantitative data analysis (meta-analyses)}

Table 1 presents unadjusted OR estimates with their 95\% CIs of attendance at breast screening for factors that were reported in three or more studies. The analyses gave $I^{2}$ values of around $99 \%$, meaning that there was a high level of heterogeneity, except for the analysis of homeowners versus tenants, where the $I^{2}$ value was $38.9 \%$ (table 1 ).

We compared the odds of attending mammographic screening by the age bands most commonly eligible for national screening programmes (60-69 and 50-59). There was no significant difference by age group in metaanalyses of observational studies $(n=16$; OR $0.97,95 \%$ CI: 
Table 1 Results of meta-analyses ${ }^{\star}$

\begin{tabular}{|c|c|c|c|}
\hline Variables & $\begin{array}{l}\text { Number of women } \\
\text { (number of studies } \\
\text { included)† }\end{array}$ & $\%$ uptake & $\begin{array}{l}\text { OR of attendance (unadjusted): } \\
\text { range | overall }(95 \% \mathrm{Cl})\end{array}$ \\
\hline \multicolumn{4}{|l|}{ Age $(60-69$ vs $50-59) \ddagger$} \\
\hline Observational studies & $5065779(16)$ & 56 vs 55 & 0.65 to $1.42 \mid 0.97$ (0.88 to 1.08$)$ \\
\hline Intervention studies & $2343(3)$ & 52 vs 57 & 0.24 to $1.16 \mid 0.78$ (0.47 to 1.31$)$ \\
\hline Rescreening studies (age at initial screen) & $271641(3)$ & 74 vs 74 & 0.93 to $1.05 \mid 0.99$ (0.93 to 1.06$)$ \\
\hline Education level & $550646(6)$ & & \\
\hline Medium vs low & & 83 vs 77 & 1.05 to $1.45 \mid 1.24$ (1.09 to 1.41$)$ \\
\hline High vs low & & 81 vs 77 & 0.76 to $1.31 \mid 1.10$ (0.97 to 1.26$)$ \\
\hline High vs medium & & 81 vs 83 & 0.61 to $1.10 \mid 0.89$ (0.78 to 1.02$)$ \\
\hline $\begin{array}{l}\text { Housing tenure (homeowner vs tenant/non- } \\
\text { owner) }\end{array}$ & $223293(3)$ & 84 vs 70 & 2.06 to $2.20 \mid 2.16$ (2.08 to 2.23$)$ \\
\hline $\begin{array}{l}\text { Country of origin (non-immigrants vs } \\
\text { immigrants) }\end{array}$ & $2409902(7)$ & 81 vs 60 & 1.75 to $2.81 \mid 2.23$ (2.00 to 2.48 ) \\
\hline Income & $1193238(5)$ & & \\
\hline Intermediate vs low & & 77 vs 66 & 1.78 to $2.09 \mid 1.96$ (1.68 to 2.29 ) \\
\hline High vs low & & 80 vs 66 & 1.61 to $2.87 \mid 2.18$ (1.86 to 2.56 ) \\
\hline High vs intermediate & & 80 vs 77 & 0.81 to $1.37 \mid 1.11$ (0.95 to 1.30$)$ \\
\hline Marital status & $1293753(7)$ & 80 vs 69 & 1.38 to $2.36 \mid 1.86$ (1.58 to 2.19 ) \\
\hline \multicolumn{4}{|l|}{$\begin{array}{l}\text { (Married/cohabiting vs unmarried/non- } \\
\text { cohabiting) }\end{array}$} \\
\hline Residence (rural vs urban) & $65641(3)$ & 74 vs 65 & 0.80 to $1.59 \mid 1.12$ (0.76 to 1.66$)$ \\
\hline $\begin{array}{l}\text { Previous result of mammogram (rescreening } \\
\text { studies only: false positive vs normal) }\end{array}$ & $3540953(6)$ & 60 vs 68 & 0.49 to $0.89 \mid 0.77$ (0.68 to 0.88$)$ \\
\hline Socioeconomic status (SES) & $6600283(11)$ & & \\
\hline Medium vs low & & 56 vs 48 & 1.08 to $2.35 \mid 1.45$ (1.20 to 1.75$)$ \\
\hline High vs low & & 54 vs 48 & 0.75 to $3.59 \mid 1.69$ (1.40 to 2.05$) \S$ \\
\hline High vs medium & & 54 vs 56 & 0.69 to $1.53 \mid 1.17$ (0.96 to 1.41$)$ \\
\hline \multicolumn{4}{|c|}{$\begin{array}{l}\text { *All results in this table are for observational studies except the data for age, which includes results for the separate meta-analysis of } \\
\text { intervention studies. } \\
\text { tReferences for studies pooled for meta-analyses of observational studies are provided in forest plots in figures } 3 \text { and } 4 \text {. } \\
\text { †We focused on the age bands most commonly eligible in population-based programmes and did not analyse odds for those younger than } \\
\text { age } 50 \text { or older than } 69 \text {. } \\
\text { §The ORs and Cls for SES include all relevant observational studies. We also performed a sensitivity analysis by removing the large study } \\
\text { from France by DeBorde et al }{ }^{18} \text { which found that women with high or medium SES were both more likely to attend compared with women of } \\
\text { lower SES (OR 1.84, } 95 \% \text { Cl: } 1.55 \text { to } 2.17, p<0.001 \text {; and OR } 1.49,95 \% \text { Cl: } 1.27 \text { to } 1.76, p<0.001, \text { respectively). }\end{array}$} \\
\hline \multicolumn{2}{|c|}{$\begin{array}{l}0.88 \text { to } 1.08, \mathrm{p}=0.631 \text {, figure } 3 \text { ) or intervention trials ( } \mathrm{n}=3 \text {; } \\
\text { OR } 0.78,95 \% \text { CI: } 0.47 \text { to } 1.31, \mathrm{p}=0.354 \text { ). } \\
\text { We grouped education data from six studies to approx- } \\
\text { imate the United Nations Educational, Scientific and } \\
\text { Cultural Organisation (UNESCO) three-level classifica- } \\
\text { tion: low ( } \leq 10 \text { years), middle ( } 11-15 \text { years) and high }(>15 \\
\text { years). Compared with women with a low level of educa- } \\
\text { tion, women with a medium level were more likely to } \\
\text { attend (OR } 1.24,95 \% \text { CI: } 1.09 \text { to } 1.41 \text {, } p<0.001 \text { ). Results } \\
\text { from comparisons of women with a high level of educa- } \\
\text { tion versus low or medium levels were not statistically } \\
\text { significant (figure } 4 \text { A). } \\
\text { The odds of attending mammographic screening were } \\
\text { higher for homeowners than for tenants or non-owners } \\
\text { (n=3; OR } 2.16,95 \% \text { CI: } 2.08 \text { to } 2.23, p<0.001 \text {, figure } 3 \text { ). }\end{array}$} & $\begin{array}{l}\text { Meta-analysis } \\
\text { that people bo } \\
\text { were more lik } \\
2.23,95 \% \text { CI: } 2 \\
\text { We meta-ana } \\
\text { SES. Data for } \\
\text { into low, med } \\
\text { medium or hi } \\
\text { those with a lo } \\
\text { CI: } 1.20 \text { to } 1.75 \\
1.40 \text { to } 2.05 \text {, p } \\
\text { (DeBorde) } \\
\text { a higher SES } \\
\text { either a low or } \\
\text { tivity analysis e }\end{array}$ & $\begin{array}{l}\text { ticipants' country of origin showed } \\
\text { he study country (non-immigrants) } \\
\text { attend than immigrants ( } \mathrm{n}=7 \text {; OR } \\
2.48, \mathrm{p}<0.001 \text {, figure } 3 \text { ). } \\
\text { attendance using two measures of } \\
\text { SES from } 11 \text { studies were grouped } \\
\text { nd high categories. Women with } \\
\text { were more likely to attend than } \\
\text { (medium vs low SES OR } 1.45,95 \% \\
01 \text {; high vs low SES OR } 1.69,95 \% \text { CI: } \\
\text { figure } 4 \mathrm{~B} \text { ). One study from France } \\
\text { nillion) reported that women with } \\
\text { ss likely to attend than those with } \\
\text { hediate SES. We conducted a sensi- } \\
\text { g that study, but it made very little }\end{array}$ \\
\hline
\end{tabular}


Table 2 Likelihood of attending screening by factors not suitable for meta-analysis in observational studies

\begin{tabular}{|c|c|c|c|c|}
\hline Variable & $\mathbf{N}^{*}$ & Included studies & $\begin{array}{l}\% \text { uptake: variable } \\
\text { vs reference } \\
\text { category }\end{array}$ & OR (95\% Cl) \\
\hline \multicolumn{5}{|l|}{ Less likely to attend } \\
\hline \multirow[t]{2}{*}{ No access to vehicle } & 144181 & Jensen $2012 b$ & 61 vs 82 & 0.33 (0.32 to 0.34$)$ \\
\hline & 37059 & O’Reilly 2012 & 60 vs 78 & 0.43 (0.41 to 0.46$)$ \\
\hline Negative attitude about breast screening & 497 & Kee 1993 & 53 vs 60 & 0.44 (0.35 to 0.55$)$ \\
\hline Receiving disability benefits & 885979 & Le 2019 & 69 vs 76 & $0.70(0.70$ to 0.71$)$ \\
\hline First invitation to screening & 742786 & Renshaw 2010 & 40 vs 76 & 0.22 (0.21 to 0.22$)$ \\
\hline \multirow[t]{2}{*}{ Spoken/preferred language not English } & 18851 & Blanchard 2004 & 62 vs 83 & 0.33 (0.28 to 0.39$)$ \\
\hline & 43819 & Tatla 2003 & 60 vs 78 & 0.43 (0.41 to 0.46$)$ \\
\hline \multirow[t]{2}{*}{ Long-term limiting illness } & 37059 & O’Reilly 2012 & 71 vs 77 & 0.71 (0.68 to 0.75$)$ \\
\hline & 144264 & Jensen $2015 b$ & 71 vs 80 & 0.64 (0.61 to 0.66$)$ \\
\hline Smoking (current) & 28874 & Katz 2018 & 84 vs 88 & 0.72 (0.65 to 0.79$)$ \\
\hline Living in crowded housing conditions & 31948 & Zackrisson 2004 & 37 vs 66 & 0.29 (0.24 to 0.36$)$ \\
\hline \multicolumn{5}{|l|}{ Employment status } \\
\hline \multirow{2}{*}{$\begin{array}{l}\text { Outside workforce vs employed/self- } \\
\text { employed }\end{array}$} & 640843 & Le 2019 & 63 vs 77 & 0.51 (0.50 to 0.51$)$ \\
\hline & 119269 & Jensen $2012 b$ & 77 vs 83 & 0.66 (0.64 to 0.68$)$ \\
\hline \multirow{2}{*}{$\begin{array}{l}\text { Unemployed vs employed/self- } \\
\text { employed }\end{array}$} & 481911 & Le 2019 & 61 vs 77 & 0.47 (0.45 to 0.49$)$ \\
\hline & 102178 & Jensen $2012 b$ & 67 vs 83 & 0.41 ( 0.40 to 0.43$)$ \\
\hline Number of childbirths & \multirow[t]{3}{*}{46041} & \multirow[t]{3}{*}{ Lagerlund 2002} & & \\
\hline 0 vs $1-2$ & & & 82 vs 91 & 0.44 (0.40 to 0.48$)$ \\
\hline $3+v s$ 1-2 & & & 90 vs 91 & 0.81 ( 0.75 to 0.87$)$ \\
\hline No family history of BC & 119502 & O’Byrne 2000 & 85 vs 86 & 0.90 (0.86 to 0.94$)$ \\
\hline Type of clinic (mobile vs fixed) & 119502 & O’Byrne 2000 & 84 vs 85 & 0.93 (0.88 to 0.98$)$ \\
\hline Schizophrenia & 110240 & Chochinov 2009 & 45 vs 58 & $0.58(0.52$ to 0.64$)$ \\
\hline \multicolumn{5}{|l|}{ More likely to attend } \\
\hline No comorbidities & 76520 & Larsen 2018 & 82 vs 75 & 1.53 (1.46 to 1.60$)$ \\
\hline $\begin{array}{l}60+\text { primary care visits during 6-year } \\
\text { study period (vs }<60)\end{array}$ & 43968 & Katz 2018 & 91 vs 79 & 2.70 (2.55 to 2.86$)$ \\
\hline Depression & 38823 & Katz 2018 & 86 vs 85 & 1.12 (1.02 to 1.23$)$ \\
\hline Good general health & 37059 & O’Reilly 2012 & 77 vs 68 & 1.55 (1.46 to 1.64$)$ \\
\hline Heart disease & 6501 & Katz 2018 & 90 vs 85 & 1.75 (1.61 to 1.91$)$ \\
\hline Not living in capital city & 885979 & Le 2019 & 76 vs 62 & 1.94 (1.91 to 1.97$)$ \\
\hline Previous attender & 11664 & $\begin{array}{l}\text { Taylor-Phillips } \\
2013\end{array}$ & 73 vs 45 & 3.32 (3.05 to 3.61$)$ \\
\hline Citizen of country & 885979 & Le 2019 & 75 vs 51 & 2.88 (2.82 to 2.94$)$ \\
\hline Member of majority racial/ethnic group & 17997 & Blanchard 2004 & 85 vs 75 & 1.70 (1.52 to 1.89$)$ \\
\hline \multicolumn{5}{|l|}{ Religion } \\
\hline Catholic vs none & 37140 & O’Reilly 2012 & 74 vs 68 & 1.40 (1.25 to 1.47$)$ \\
\hline Protestant vs none & & O’Reilly 2012 & 77 vs 68 & 1.57 (1.46 to 1.70$)$ \\
\hline Never HRT use & 119502 & O’Byrne 2000 & 16 vs 14 & 1.13 (1.09 to 1.17$)$ \\
\hline Referral by health professional & 56420 & Tatla 2003 & 77 vs 76 & 1.05 (1.00 to 1.10$)$ \\
\hline \multicolumn{5}{|c|}{ No difference in attendance or mixed results } \\
\hline $\mathrm{BMI}$ & 19168 & Katz 2018 & 87 vs 87 & 0.95 (0.87 to 1.04$)$ \\
\hline$>0$ GPs per 100000 inhabitants & 4865 & Pornet 2010 & 55 vs 56 & 0.96 (0.85 to 1.08$)$ \\
\hline$>0$ radiologists per 100000 inhabitants & 4865 & Pornet 2010 & 52 vs 56 & 0.87 (0.72 to 1.05$)$ \\
\hline
\end{tabular}


Table 2 Continued

\begin{tabular}{|c|c|c|c|c|}
\hline Variable & $\mathbf{N}^{*}$ & Included studies & $\begin{array}{l}\% \text { uptake: variable } \\
\text { vs reference } \\
\text { category }\end{array}$ & OR (95\% Cl) \\
\hline Diabetes & 504288 & Chan 2014 & 60 vs 66 & 0.79 (0.78 to 0.80$)$ \\
\hline Distance to screening centre & 137419 & Jensen 2012b & 77 vs 80 & $0.86(0.84$ to 0.88$)$ \\
\hline Physician years since graduation & 105575 & Makedonov 2015 & 74 vs 75 & 1.03 (0.99 to 1.06$)$ \\
\hline
\end{tabular}

${ }^{*}$ Reflects the number of participants analysed for each factor, which can differ for different factors in the same study depending on data availability.

BMI, body mass index; GPs, general practitioners; HRT, hormone replacement therapy.

difference to the odds of attending: women with high or medium SES were both more likely to attend compared with women of lower SES (OR 1.84, 95\% CI: 1.55 to 2.17, $\mathrm{p}<0.001$, and OR $1.49,95 \%$ CI: 1.27 to $1.76, \mathrm{p}<0.001$, respectively).

Data on income from five studies were grouped into low, intermediate and high categories. Women with an intermediate or high income were more likely to attend than those with low income (intermediate vs low income OR $1.96,95 \%$ CI: 1.68 to $2.29, \mathrm{p}<0.001$; high vs low OR 2.18, $95 \%$ CI: 1.86 to $2.56, \mathrm{p}<0.001$; high vs intermediate OR $1.11,95 \%$ CI: 0.95 to $1.30, p=0.20$, figure $4 \mathrm{C}$ ). For both income and SES, there was no significant difference between women at intermediate and high levels, indicating that there was no statistically significant dose response effect for higher SES or income.

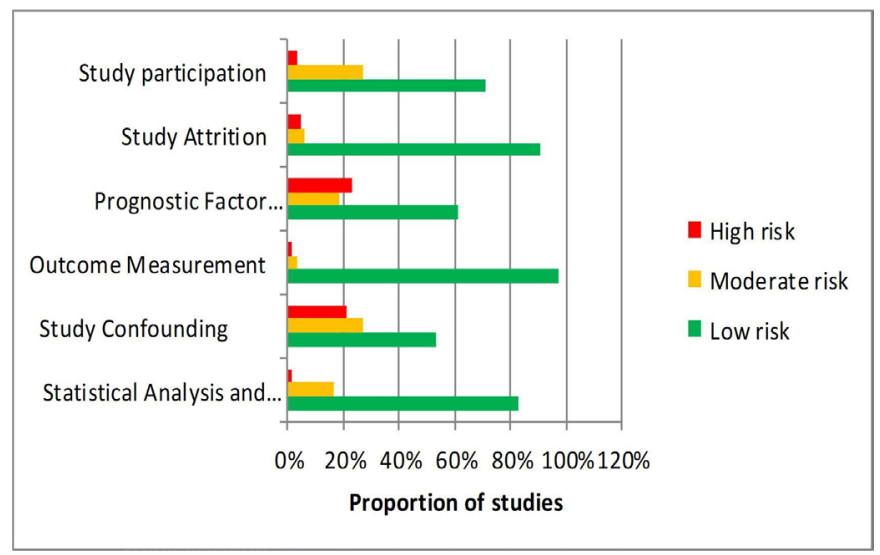

Figure 2 Overall summary of QUIPS risk of bias scores: risk of bias (RoB) of all included studies was appraised by two independent reviewers using the Quality in Prognosis Studies (QUIPS) tool. The QUIPS tool covers six RoB domains (participation, attrition, prognostic factor, confounding factors, outcome measurement and analysis and reporting), each of which includes multiple items that are judged separately. A conclusive judgement for each RoB domain is reached and expressed on a three-grade scale (high, moderate or low RoB). RoB across studies was generally low on all domains.
Women who were married or cohabiting were more likely to attend than their unmarried or non-cohabiting counterparts ( $\mathrm{n}=7$; OR $1.86,95 \%$ CI: 1.58 to 2.19 , $\mathrm{p}<0.001$, figure 3).

We analysed data separately for studies with samples made up only of women who had previously attended mammographic screening (ie, rescreening studies). Six of these studies reported data on attendance based on the results of a previous mammogram. Women who had previously received a false-positive result were less likely to attend than those with a normal result (OR 0.78, 95\% CI: 0.68 to $0.88, \mathrm{p}<0.001$, figure 3 ).

There was no statistically significant difference in attendance among women living in rural compared with urban areas $(\mathrm{n}=3$; OR $1.12,95 \%$ CI: 0.76 to $1.66, \mathrm{p}=0.557)$.

\section{Narrative synthesis}

Factors that could not be meta-analysed (because they were reported in fewer than three studies or could not be pooled) are reported in table 2 with ORs.

These studies include a variety of factors associated with reduced attendance clustered around sociodemographic, accessibility and logistics (living in crowded housing and being unemployed, receiving disability benefits, lack of access to a vehicle), and spoken language not English.

Associations with women's health status, behaviours, attitudes and knowledge showed a mixed picture. There was some evidence that good general health, lack of comorbidity and not taking hormone replacement therapy (HRT) were all associated with higher attendance, but studies also reported higher attendance among women with a higher numbers of previous clinic visits, depression and heart disease. A previous negative attitude to breast screening, limiting long-term illness, schizophrenia, nonwork-related stress and current smoking were associated with lower attendance.

Factors that did not show any statistical difference included body mass index and service provision factors. No difference in women's attendance was found according to availability of general practitioners or radiologists or physician years since graduation, and there were 


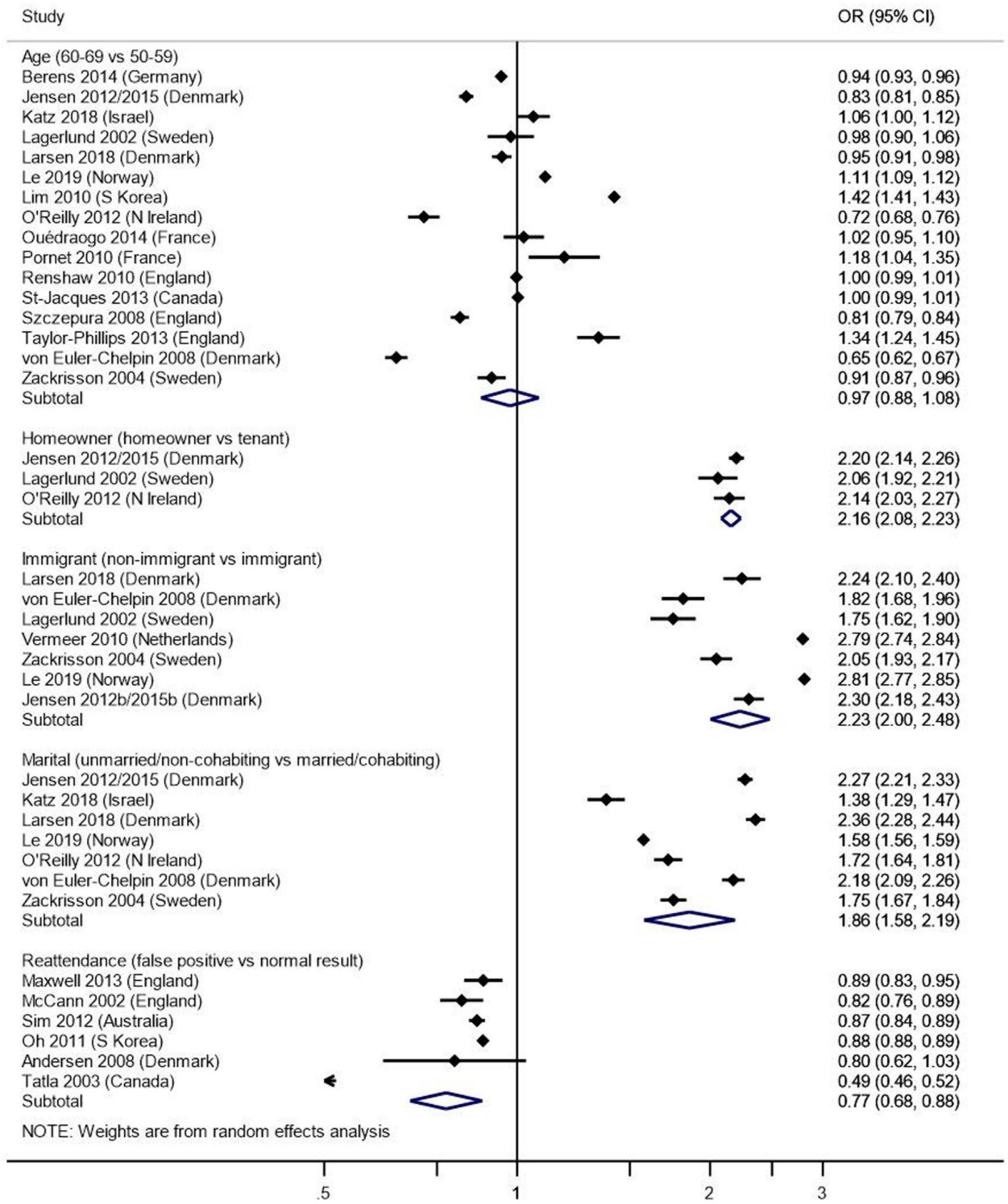

Figure 3 Meta-analyses. This figure shows comparisons of the odds of attending mammographic screening, using randomeffects analysis, in observational studies by the following variables. Points to the left of the centre line $(<1)$ suggest a lower likelihood of attending screening, while points to the right of the centre line $(>1)$ indicate a higher likelihood of attending. Age bands: we compared the age bands most commonly eligible for national screening programmes (60-69 and 50—59); there was no significant difference by age group ( $n=16$; OR $0.97,95 \% \mathrm{Cl}$ : 0.88 to $1.08, p=0.631$ ); Home ownership: we compared people who own their homes to those who are tenants or do not own their homes; the odds of attending were higher for homeowners than for tenants or non-owners ( $\mathrm{n}=3$; OR 2.16, 95\% Cl: 2.08 to 2.23, $\mathrm{p}<0.001$ ); Immigrant status: we compared screening attendance of people born in the country in which the study took place (non-immigrants) to those born in another country (immigrants); non-immigrants were more likely to attend than immigrants ( $n=7$; OR $2.23,95 \%$ Cl: 2.00 to $2.48, p<0.001$ ). Marital status: we compared women who were married or cohabiting to those who were unmarried or not cohabiting: women where were married/cohabiting were more likely to attend than their unmarried/non-cohabiting counterparts $(n=7 ;$ OR $1.86,95 \%$ Cl: 1.58 to $2.19, p<0.001)$. Reattendance; using data from studies with samples made up only of women who had previously attended mammographic screening, we compared women who had previously received a false-positive to those who had had a normal result; those with a previous false-positive result were less likely to reattend (OR $0.78,95 \% \mathrm{Cl}: 0.68$ to $0.88, p<0.001)$.

mixed results according to distance to screening centre and diabetes.

\section{DISCUSSION}

We undertook a comprehensive review of the current evidence on patient-level factors associated with breast cancer (mammographic) screening attendance. Where appropriate, meta-analyses were performed to determine the strength of association.

\section{Main findings}

In line with other systematic reviews, we found that in general higher SES status, higher income,${ }^{14}$ being born in the country of residence (ie, non-immigrant) ${ }^{12}$ and home ownership (compared with renting) predicted 


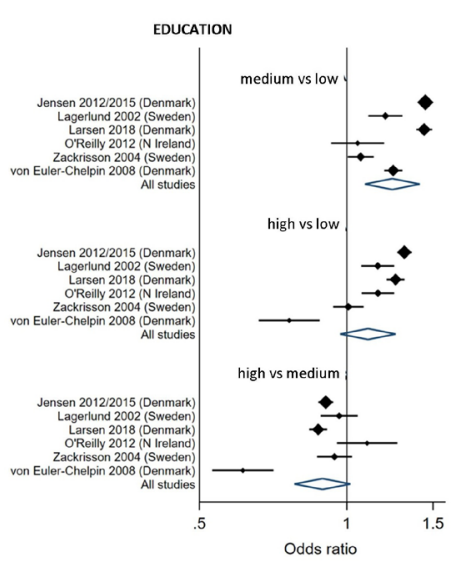

4A. Meta-analysis of attendance by educational

level

Medium vs low level: OR 1.24, 95\%Cl: 1.09-1.41,

Medium vs

$p<0.001$
High vs low level: OR $1.10,95 \% \mathrm{Cl}: 0.97-1.26$,

High vs lon

$p=0.133$

High vs medium level: $\mathrm{OR} 0.89,95 \% \mathrm{Cl}: 0.78$

$1.02, p=0.12$

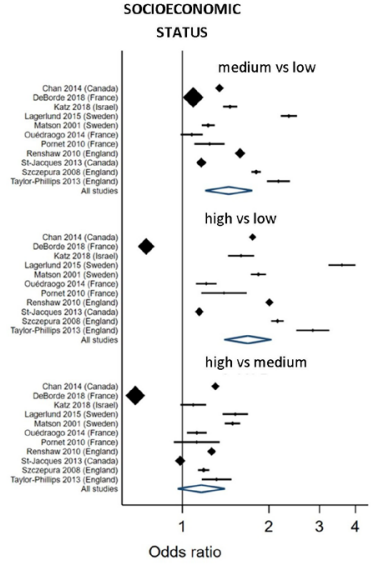

4B. Meta-analysis of attendance by socioeconomic status (SES) Medium vs low level: OR $1.45,95 \% \mathrm{Cl}$ : 1.20-1.75, $\mathrm{p}<0.001$

High vs low level: OR $1.69,95 \%$ Cl: 1.40 High vs low level

$2.05, \mathrm{p}<0.001$
High vs medium level: $\mathrm{OR} 1.17,95 \% \mathrm{Cl}$ High vs medium leve

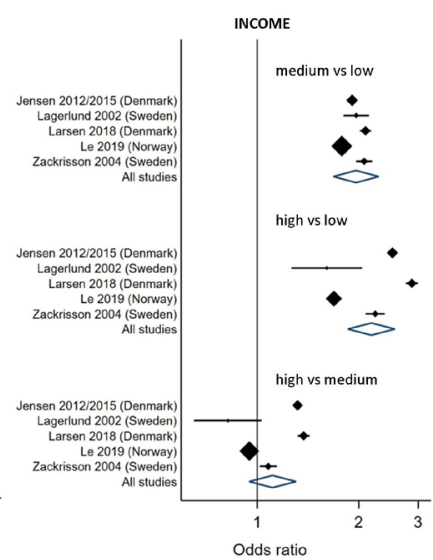

4C. Meta-analysis of attendance by

income

Medium vs low level: OR 1.96, 95\%Cl: 1.68

$-2.29, p<0.001$

High vs low level: $O R 2.18,95 \% \mathrm{Cl}: 1.86$

.56, $p<0.001$

$2.56, p<0.001$ : OR $2.18,95 \% \mathrm{Cl:} 1.86-$

$0.95-1.30, p=0.20$

Figure 4 Meta-analyses of attendance by educational level, socioeconomic status (SES) and income. These figures show random-effects meta-analyses of screening attendance by educational level and socioeconomic status in observational studies. Points to the left of the centre line $(<1)$ suggest a lower likelihood of attending screening, while points to the right of the centre line $(>1)$ indicate a higher likelihood of attending. Figure $4 \mathrm{~A}$ shows the effects of different levels of education on screening attendance. We grouped education data to approximate the United Nations Educational, Scientific and Cultural Organization (UNESCO) three-level classification: low ( $\leq 10$ years), middle (11-15 years) and high (>15 years). Compared with women with a low level of education, women with a medium level were more likely to attend (OR 1.24, 95\% $\mathrm{Cl}$ : 1.09 to $1.41, \mathrm{p}<0.001$ ). Results from comparisons of women with a high level of education versus low or medium levels were not statistically significant (figure 4A). Figure 4B shows the meta-analysis of attendance by overall SES. Studies were grouped into low, medium and high categories. Women with medium or high SES were more likely to attend than those with a low SES (medium vs low SES OR 1.45, 95\% Cl: 1.20 to $1.75, \mathrm{p}<0.001$; high vs low SES OR 1.69, 95\% Cl: 1.40 to 2.05, $\mathrm{p}<0.001$, figure 4B). Figure 4C shows the meta-analysis of screening attendance by income. Studies were grouped into low, intermediate and high categories. Women with an intermediate or high income were more likely to attend than those with low income (intermediate vs low income OR 1.96, $95 \% \mathrm{Cl}$ : 1.68 to $2.29, \mathrm{p}<0.001$; high vs low OR 2.18, 95\% Cl: 1.86 to 2.56, $\mathrm{p}<0.001$; high vs intermediate OR 1.11 , $95 \% \mathrm{Cl}: 0.95$ to $1.30, \mathrm{p}=0.20$, figure $4 \mathrm{C}$ ). For both income and SES, there was no significant difference between women at intermediate and high levels, indicating that there was no statistically significant dose response effect for higher SES or income.

mammographic screening attendance. However, it appears that women with a higher SES or income were not more likely to attend than those with an intermediate level. We hypothesise that women with a higher SES may be more likely to use alternative screening services (ie, opportunistic or privately funded screening) compared with women with a low or intermediate SES, thus their attendance would not be apparent in studies using data from national screening programmes. This was suggested as a limitation by many of the included studies in this review, most notably the large study from France ${ }^{18}(n=4.8$ million), which was the only study to find that women with a higher SES were less likely to attend than those with either a low or intermediate SES. The authors of that study note the high levels of opportunistic screening available to women with a high SES in France. We conducted a sensitivity analysis excluding that study, but it made very little difference to the ORs for attendance.

A medium level of education was also associated with screening attendance when compared with a low level, but a higher level of education was not associated with increased attendance compared with either medium or lower levels. As with the analyses of SES, it is possible that women with the highest levels of education are more likely to use alternative screening services not reflected in data from public screening programmes.

We hypothesised that some variation in relation to education or SES might be due to changes in women's attitudes to breast screening as a result of concerns about its overall benefits, ${ }^{65} 88$ perhaps related to the informedchoice agenda. ${ }^{4}$ However, we found no population screening studies investigating this.

Our results also support previous research indicating that marital status is associated with attendance at mammography, ${ }^{65}$ 88-91 with women who were married or cohabiting more likely to attend than their unmarried or non-cohabiting counterparts. Previous literature indicates lower uptake among women from minority-ethnic backgrounds. ${ }^{92} 93$ While our data were not sufficient to meta-analyse ethnicity, we did find that immigrant women were less likely to attend screening than non-immigrants.

We did not find a significant effect of age. There was very high heterogeneity here, with individual large studies finding highly statistically significant results in both directions. We hypothesised that attendance may be higher among older women because they have been invited 
to breast screening for at least two decades, and attendance may have become more routine in this cohort, and possibly less likely to be affected by recent debates around the risks and benefits of screening. To explore this, we did a post-hoc analysis of the effect of age on attendance by the year of study completion. We found that older women were more likely to attend compared with younger women in more recent studies (ie, those completed since 2010), but that the opposite was true in older studies, particularly those published before 2005 .

Women who received a false-positive result at a previous screening were less likely to attend than those with a normal result, confirming previous findings. ${ }^{94}$

\section{Strengths and limitations}

This review has many strengths. The large number of studies included $(\mathrm{n}=66)$, involving more than 22 million women, represents a comprehensive overview of available evidence. Studies included in the meta-analysis were judged to have a low RoB on most domains and included large numbers of women. At least two reviewers were involved at all stages to reduce the risk of errors and bias. This study was undertaken from the perspective of population-based breast cancer screening programmes and we were strict in our eligibility criteria in including only those studies. Studies where the sampling frame was restricted to population subgroups (and not based on population-based screening programmes) were excluded. We also excluded studies that relied on selfreported attendance (though it is important to note that self-report is essential for some factors, such as ethnicity and attitudes to screening).

A limitation is that most studies reported cross-sectional attendance data, which included mixed groups of those who were attending for the first time and some who had previously attended. Also, we inevitably had to make choices of categories for meta-analysis which may affect meta-analytic results; where possible we used independent sources to select appropriate categorisations.

The main limitation of this review is significant betweenstudy heterogeneity. Although we used random-effect models throughout, our results should be considered in light of this. We chose random-effects models as almost all of our analyses contained heterogeneity and it is also expected that there would be differences in attendance across the different study populations. Studies with larger sample sizes are assumed to contain the least uncertainty and are given higher weightings than smaller studies. For analyses of small numbers of studies, the random-effects analysis may struggle to correctly estimate uncertainty, but any meta-analysis performed on few studies would have its limitations, and the use of random-effects analysis maintained consistency with the other analyses.

Heterogeneity may in part be due to differences between health systems and the organisation of mammographic screening, as well as differences in the culture and attitudes of the populations served. We conducted sensitivity analysis to determine the impact of a very large study with an extreme effect size ${ }^{18}$ on the meta-analysis of SES. For some outcomes (such as age), the heterogeneity encompasses studies with highly significant results in both directions, and here the results of the meta-analysis should be interpreted with great caution. For other variables (such as reattendance after false-positive results), the high $I^{2}$ simply reflects that there were very large studies with very small CIs, which all had point estimates of different magnitude in the same direction. Here the meta-analysis results show a consistent effect, with some disagreement between studies on the exact size of effect.

Another limitation is that we extracted univariable associations with uptake. In practice, many of the variables investigated will be highly correlated, and there will be complex interactions and confounding which we have not been able to account for. While some studies did report multivariable models, these were varied in structure, methods and variables included, so would have been difficult to combine in any meaningful way. We were therefore unable to undertake multivariable meta-regression analysis, examining the effects of individual attendance factors on overall attendance.

For the studies included in the narrative analysis, large numbers of women were also often involved, but these studies should be treated with caution as they are potentially subject to bias. The risk of confounding was found to be high in these studies using the QUIPs tool. However, confounding is inherent in the design of populationbased observational and especially ecological designs.

To investigate the risk of reporting bias, we conducted funnel plots (online supplemental file F), which demonstrated the high level of heterogeneity present between the studies in our analyses. Age was the only analysis where the studies disagree over the direction of attendance, however the disagreement is among larger studies, suggesting this is unlikely to be associated with biased reporting and instead down to the study heterogeneity. All other analyses, while having studies which disagree on the point estimate, have agreement as to which group is more or less likely to attend mammographic screening. Overall, we are not concerned about reporting bias.

Finally, we have not included health insurance (or lack of health insurance) as a factor in the narrative analysis because of the problems of comparison between countries.

\section{CONCLUSIONS}

A wide variety of factors affect a woman's decision to attend breast screening. Our main findings are that attendance was lower in women with lower SES, those who were immigrants, non-homeowners and those with previous false-positive results. Based on our current findings, if screening programmes wish to improve equity of access to breast screening services, they should concentrate on women facing access (practical, physical, psychological and financial) barriers. 
Future research in this area would also need to systematically assess the effects of interventions to reduce the impact of access barriers to screening attendance.

\section{Deviations from study protocol}

To assess RoB, the QUIPS tool was used rather than the Quality Assessment Tool; and for data synthesis, despite significant heterogeneity, meta-analysis was possible for some predictors. In addition, we clarified our inclusion criteria to include only studies with data from routine population-based mammography screening programmes in order to ensure generalisability.

\section{Twitter Wendy Lynn Knerr @warwickmed}

Acknowledgements The authors would like to thank Magdalena Skrybant, Patient and Public Involvement and Engagement Lead, Applied Research Centre West Midlands (formerly Centres for Leadership in Applied Health and Care, West Midlands) for her support in coordinating public engagement in this project.

Contributors RM conceived the study as part of her PhD Dissertation, and it was further refined in collaboration with AC and ST-P. AC, CS and WLK further developed the inclusion and exclusion criteria. SJ undertook database searches and $A C, H F$, RM, LA-K, SW and WLK reviewed titles and abstracts. Each study retained for full-text review was reviewed by RM and WLK. Discrepancies regarding inclusion and exclusion were resolved by AC and CS. RM and WLK did data extraction, and data were checked by OAU and CN. Studies were critically appraised by AA, AT, CS and WLK. Meta-analyses were conducted by DG. Thematic synthesis was done by AC, ST-P and WLK. All authors contributed to the manuscript and approved the final version. $A C$ is the guarantor for this paper.

Funding This research was funded by the National Institute for Health Research Collaboration for Leadership in Applied Health Research and Care West Midlands (NIHR CLAHRC WM), now recommissioned as NIHR Applied Research Collaboration West Midlands (NIHR ARC WM). RM, L A-K, ST-P, SW, HF, CS and AC were all supported by the NIHR CLAHRC WM and WLK, AA, AC and L-AK are all partly supported by the NIHR ARC WM. OAU is supported by the NIHR using Official Development Assistance (ODA) funding. ST-P is supported by an NIHR Career Development Fellowship (CDF-2016-09-018). The views expressed in this publication are those of the author(s) and not necessarily those of the UK National Health Service (NHS), the NIHR or the Department of Health and Social Care.

Competing interests RM reports personal fees from the National Institute for Health Research (NIHR) Centre for Leadership in Applied Research and Health Care (CLARHC) West Midlands during the conduct of the study. LA-K reports grants from the NIHR during the conduct of the study. ST-P reports grants from NIHR outside the submitted work. AC reports grants from the NIHR for the NIHR Applied Research Centre (ARC) West Midlands and previously from the NIHR CLARHC West Midlands, which supported her and researchers working on this project. AC also received grants from Public Health England (PHE) outside the submitted work.

Patient consent for publication Not required.

Provenance and peer review Not commissioned; externally peer reviewed.

Data availability statement № data are available. No new data have been created in the preparation of this report and therefore there is nothing available for access and further sharing. All queries should be submitted to the corresponding author.

Supplemental material This content has been supplied by the author(s). It has not been vetted by BMJ Publishing Group Limited (BMJ) and may not have been peer-reviewed. Any opinions or recommendations discussed are solely those of the author(s) and are not endorsed by BMJ. BMJ disclaims all liability and responsibility arising from any reliance placed on the content. Where the content includes any translated material, BMJ does not warrant the accuracy and reliability of the translations (including but not limited to local regulations, clinical guidelines, terminology, drug names and drug dosages), and is not responsible for any error and/or omissions arising from translation and adaptation or otherwise.

Open access This is an open access article distributed in accordance with the Creative Commons Attribution 4.0 Unported (CC BY 4.0) license, which permits others to copy, redistribute, remix, transform and build upon this work for any purpose, provided the original work is properly cited, a link to the licence is given, and indication of whether changes were made. See: https://creativecommons.org/ licenses/by/4.0/.

\section{ORCID iDs}

Wendy Lynn Knerr http://orcid.org/0000-0002-6475-9417

Daniel Gallacher http://orcid.org/0000-0003-0506-9384

Abimbola Ayorinde http://orcid.org/0000-0002-4915-5092

Olalekan A Uthman http://orcid.org/0000-0002-8567-3081

Sian Taylor-Phillips http://orcid.org/0000-0002-1841-4346

\section{REFERENCES}

1 Sung H, Ferlay J, Siegel RL, et al. Global cancer statistics 2020: GLOBOCAN estimates of incidence and mortality worldwide for 36 cancers in 185 countries. CA Cancer J Clin 2021;71:209-49.

2 Malvezzi M, Carioli G, Bertuccio P, et al. European cancer mortality predictions for the year 2019 with focus on breast cancer. Ann Oncol 2019;30:781-7.

3 Marmot MG, Altman DG, Cameron DA, et al. The benefits and harms of breast cancer screening: an independent review. Br J Cancer 2013;108:2205-40.

4 Martínez-Alonso M, Carles-Lavila M, Pérez-Lacasta MJ, et al. Assessment of the effects of decision AIDS about breast cancer screening: a systematic review and meta-analysis. BMJ Open 2017;7:e016894.

5 Reder M, Berens E-M, Spallek J, et al. Development of the informed choice in mammography screening questionnaire (IMQ): factor structure, reliability, and validity. BMC Psychol 2019;7:17.

6 Coyle C, Kinnear H, Rosato M, et al. Do women who intermittently attend breast screening differ from those who attend every invitation and those who never attend? J Med Screen 2014;21:98-103.

7 Ross NA, Rosenberg MW, Pross DC, et al. Contradictions in women's health care provision: a case study of attendance for breast cancer screening. Soc Sci Med 1994;39:1015-25.

8 Aarts MJ, Voogd AC, Duijm LEM, et al. Socioeconomic inequalities in attending the mass screening for breast cancer in the south of the Netherlands--associations with stage at diagnosis and survival. Breast Cancer Res Treat 2011;128:517-25.

9 Ackerson K, Preston SD. A decision theory perspective on why women do or do not decide to have cancer screening: systematic review. J Adv Nurs 2009;65:1130-40.

10 Crosby R. Predictors of uptake of screening mammography. Coventry: University of Warwick, 2018.

11 Schueler KM, Chu PW, Smith-Bindman R. Factors associated with mammography utilization: a systematic quantitative review of the literature. J Womens Health 2008;17:1477-98.

12 Bhargava S, Moen K, Qureshi SA, et al. Mammographic screening attendance among immigrant and minority women: a systematic review and meta-analysis. Acta Radiol 2018;59:1285-91.

13 Mathioudakis AG, Salakari M, Pylkkanen L, et al. Systematic review on women's values and preferences concerning breast cancer screening and diagnostic services. Psychooncology 2019;28:939-47.

14 Smith D, Thomson K, Bambra C, et al. The breast cancer paradox: a systematic review of the association between area-level deprivation and breast cancer screening uptake in Europe. Cancer Epidemiol 2019;60:77-85.

15 Crosby R, Williamson S, Stinton C. A systematic review to identify the worldwide predictors of breast screening uptake prospero International prospective register of systematic reviews. University of York, 2016. http://www.crd.york.ac.uk/PROSPERO/display_record. asp?ID=CRD42016051597

16 Hayden JA, van der Windt DA, Cartwright JL, et al. Assessing bias in studies of prognostic factors. Ann Intern Med 2013;158:280-6.

17 Valentine J, Pigott T, Rothstein H. How many studies do you need? A primer on statistical power for meta-analysis. Journal of Educational and Behavioural Statistics 2010;35:215-47.

18 Deborde T, Chatignoux E, Quintin C, et al. Breast cancer screening programme participation and socioeconomic deprivation in France. Prev Med 2018;115:53-60.

19 Liberati A, Altman DG, Tetzlaff J, et al. The PRISMA statement for reporting systematic reviews and meta-analyses of studies that evaluate health care interventions: explanation and elaboration. PLoS Med 2009;6:e1000100.

20 Allgood PC, Maxwell AJ, Hudson S, et al. A randomised trial of the effect of postal reminders on attendance for breast screening. $\mathrm{Br} J$ Cancer 2016;114:171-6.

21 Andersen SB, Vejborg I, von Euler-Chelpin M. Participation behaviour following a false positive test in the Copenhagen mammography screening programme. Acta Oncol 2008;47:550-5. 
22 Barlow WE, Beaber EF, Geller BM, et al. Evaluating screening participation, follow-up, and outcomes for breast, cervical, and colorectal cancer in the PROSPR Consortium. J Natl Cancer Inst 2020;112:238-46.

23 Berens E-M, Stahl L, Yilmaz-Aslan Y, et al. Participation in breast cancer screening among women of Turkish origin in Germany - a register-based study. BMC Womens Health 2014;14:24.

24 Blanchard K, Colbert JA, Puri D, et al. Mammographic screening: patterns of use and estimated impact on breast carcinoma survival. Cancer 2004;101:495-507.

25 Bourmaud A, Soler-Michel P, Oriol M, et al. Decision aid on breast cancer screening reduces attendance rate: results of a large-scale, randomized, controlled study by the DECIDEO group. Oncotarget 2016:7:12885-92.

26 Chiarelli AM, Moravan V, Halapy E, et al. False-Positive result and reattendance in the Ontario breast screening program. J Med Screen 2003:10:129-33.

27 Douglas E, Waller J, Duffy SW, et al. Socioeconomic inequalities in breast and cervical screening coverage in England: are we closing the gap? J Med Screen 2016;23:98-103.

28 Finney Rutten LJ, Ebbert JO, Jacobson DJ, et al. Changes in U.S. preventive services Task force recommendations: effect on mammography screening in Olmsted County, Mn 2004-2013. Prev Med 2014;69:235-8.

29 Gatrell A, Garnett S, Rigby J, et al. Uptake of screening for breast cancer in South Lancashire. Public Health 1998;112:297-301.

30 Goldzahl L, Hollard G, Jusot F. Increasing breast-cancer screening uptake: a randomized controlled experiment. $J$ Health Econ 2018:58:228-52.

31 Hyndman JCG, Holman CDJ, Dawes VP. Effect of distance and social disadvantage on the response to invitations to attend mammography screening. J Med Screen 2000;7:141-5.

32 Jensen LF, Pedersen AF, Andersen B, et al. Identifying specific non-attending groups in breast cancer screening--population-based registry study of participation and socio-demography. BMC Cancer 2012;12:518.

33 Jensen LF, Pedersen AF, Andersen B, et al. Non-Participation in breast cancer screening for women with chronic diseases and multimorbidity: a population-based cohort study. BMC Cancer 2015;15:798

34 Katz D, Tengekyon AJ, Kahan NR, et al. Patient and physician characteristics affect adherence to screening mammography: a population-based cohort study. PLoS One 2018;13:e0194409.

35 Kee F, Telford AM, Donaghy P, et al. Enhancing mammography uptake: who do women listen to? Eur J Cancer Prev 1993;2:37-42.

36 Lagerlund M, Maxwell AE, Bastani R, et al. Sociodemographic predictors of non-attendance at invitational mammography screening-a population-based register study (Sweden). Cancer Causes Control 2002;13:73-82.

37 Lagerlund M, Merlo J, Vicente RP, et al. Does the neighborhood area of residence influence Non-Attendance in an urban mammography screening program? A multilevel study in a Swedish City. PLoS One 2015;10:e0140244

38 Lagerlund M, Sontrop JM, Zackrisson S. Psychosocial factors and attendance at a population-based mammography screening program in a cohort of Swedish women. BMC Womens Health 2014;14:33.

39 Larsen SH, Virgilsen LF, Kristiansen BK, et al. Strong association between cervical and breast cancer screening behaviour among Danish women; a register-based cohort study. Prev Med Rep 2018;12:349-54.

40 Le M, Hofvind S, Tsuruda K, et al. Lower attendance rates in BreastScreen Norway among immigrants across all levels of sociodemographic factors: a population-based study. J Public Health 2019;27:229-40.

41 Leung J, Macleod C, McLaughlin D, et al. Screening mammography uptake within Australia and Scotland in rural and urban populations. Prev Med Rep 2015;2:559-62.

$42 \mathrm{Lim}$ SM, Lee H-Y, Choi KS, et al. Trends of mammography use in a national breast cancer screening program, 2004-2008. Cancer Res Treat 2010;42:199-202.

43 Luckmann R, Costanza ME, White MJ, et al. A 4-year randomized trial comparing three outreach interventions to promote screening mammograms. Transl Behav Med 2019;9:328-35.

44 Makedonov I, Gu S, Paszat LF, et al. Organized breast screening improves reattendance compared to physician referral: a case control study. BMC Cancer 2015;15:315.

45 Matson S, Andersson I, Berglund G, et al. Nonattendance in mammographic screening: a study of intraurban differences in Malmö, Sweden, 1990-1994. Cancer Detect Prev 2001;25:132-7.
46 Maxwell AJ, Beattie C, Lavelle J, et al. The effect of false positive breast screening examinations on subsequent attendance: retrospective cohort study. J Med Screen 2013;20:91-8.

47 Mayer JA, Lewis EC, Slymen DJ, et al. Patient reminder letters to promote annual mammograms: a randomized controlled trial. Prev Med 2000;31:315-22.

48 McCann J, Stockton D, Godward S. Impact of false-positive mammography on subsequent screening attendance and risk of cancer. Breast Cancer Res 2002;4:R11.

49 Meldrum P, Turnbull D, Dobson HM, et al. Tailored written invitations for second round breast cancer screening: a randomised controlled trial. J Med Screen 1994;1:245-8.

50 Merrick EL, Hodgkin D, Horgan CM, et al. Testing novel patient financial incentives to increase breast cancer screening. Am J Manag Care 2015;21:771-9.

51 Moss SM, Brown J, Garvican L, et al. Routine breast screening for women aged 65-69: results from evaluation of the demonstration sites. Br J Cancer 2001;85:1289-94.

52 O'Byrne A-M, Kavanagh AM, Ugoni A, et al. Predictors of nonattendance for second round mammography in an Australian mammographic screening programme. J Med Screen 2000;7:190-4.

53 Offman J, Wilson M, Lamont M, et al. A randomised trial of weekend and evening breast screening appointments. Br J Cancer 2013;109:597-602.

54 Oh D, Jung DW, Jun JK, et al. On-schedule mammography rescreening in the National cancer screening program for breast cancer in Korea. Asian Pac J Cancer Prev 2011;12:2865-70.

55 Ore L, Hagoel L, Shifroni G, et al. Compliance with mammography screening in Israeli women: the impact of a pre-scheduled appointment and of the letter-style. Isr J Med Sci 1997;33:103-11.

56 O'Reilly D, Kinnear H, Rosato M, et al. Using record linkage to monitor equity and variation in screening programmes. BMC Med Res Methodol 2012;12:59.

57 Ouédraogo S, Dabakuyo-Yonli TS, Roussot A, et al. European transnational ecological deprivation index and participation in population-based breast cancer screening programmes in France. Prev Med 2014;63:103-8.

58 Peeters PH, Beckers CG, Hogervorst JM, et al. Effect on breast cancer screening response in the Netherlands of Inviting women for an additional scientific investigation. J Epidemiol Community Health 1994:48:175-7.

59 Pelfrene E, Bleyen L, De Backer G. Uptake in breast cancer screening. A sociogeographical analysis. The European Journal of Public Health 1998;8:146-9.

60 Pinckney RG, Geller BM, Burman M, et al. Effect of false-positive mammograms on return for subsequent screening mammography. Am J Med 2003:114:120-5.

61 Pornet C, Dejardin O, Morlais F, et al. Socioeconomic and healthcare supply statistical determinants of compliance to mammography screening programs: a multilevel analysis in Calvados, France. Cancer Epidemiol 2010;34:309-15.

62 Renshaw C, Jack RH, Dixon S, et al. Estimating attendance for breast cancer screening in ethnic groups in London. BMC Public Health 2010:10:157.

63 Richards SH, Bankhead C, Peters TJ, et al. Cluster randomised controlled trial comparing the effectiveness and cost-effectiveness of two primary care interventions aimed at improving attendance for breast screening. J Med Screen 2001;8:91-8.

64 Rodriguez C, Plasencia A, Schroeder DG. Predictive factors of enrollment and adherence in a breast cancer screening program in Barcelona (Spain). Soc Sci Med 1995;40:1155-60.

65 Scaf-Klomp W, van Sonderen FL, Stewart R, et al. Compliance after 17 years of breast cancer screening. J Med Screen 1995;2:195-9.

66 Segnan N, Senore C, Giordano L, et al. Promoting participation in a population screening program for breast and cervical cancer: a randomized trial of different invitation strategies. Tumori 1998;84:348-53.

67 Sim MJH, Siva SP, Ramli IS, et al. Effect of false-positive screening mammograms on rescreening in Western Australia. Med J Aust 2012;196:693-5.

68 Simon MS, Gimotty PA, Moncrease A, et al. The effect of patient reminders on the use of screening mammography in an urban health department primary care setting. Breast Cancer Res Treat 2001;65:63-70.

69 St-Jacques S, Philibert MD, Langlois A, et al. Geographic access to mammography screening centre and participation of women in the Quebec breast cancer screening programme. J Epidemiol Community Health 2013;67:861-7.

70 Sutradhar R, Gu S, Glazier RH, et al. The association between visiting a primary care provider and uptake of periodic mammograms as women get older. J Med Screen 2016;23:83-8. 
71 Szczepura A, Price C, Gumber A. Breast and bowel cancer screening uptake patterns over 15 years for UK South Asian ethnic minority populations, corrected for differences in socio-demographic characteristics. BMC Public Health 2008;8:346.

72 Taplin SH, Anderman C, Grothaus L, et al. Using physician correspondence and postcard reminders to promote mammography use. Am J Public Health 1994;84:571-4.

73 Tatla RK, Paszat LF, Bondy SJ, et al. Socioeconomic status \& returning for a second screen in the Ontario breast screening program. Breast 2003;12:237-46.

74 Taylor V, Thompson B, Lessler D, et al. A clinic-based mammography intervention targeting inner-city women. J Gen Intern Med 1999;14:104-11.

75 Taylor-Phillips S, O'Sullivan E, Kearins O, et al. The effects of a UK review of breast cancer screening on uptake: an observational before/after study. J Med Screen 2013;20:86-90.

76 Vermeer B, Van den Muijsenbergh METC. The attendance of migrant women at the National breast cancer screening in the Netherlands 1997-2008. Eur J Cancer Prev 2010;19:195-8.

77 Vidal C, Garcia M, Benito L, et al. Use of text-message reminders to improve participation in a population-based breast cancer screening program. J Med Syst 2014;38:118.

78 Visser O, van Peppen AM, Ory FG, et al. Results of breast cancer screening in first generation migrants in northwest Netherlands. Eur $J$ Cancer Prev 2005;14:251-5.

79 von Euler-Chelpin M, Olsen AH, Njor S, et al. Socio-Demographic determinants of participation in mammography screening. Int $\mathrm{J}$ Cancer 2008;122:418-23.

80 Wilf-Miron R, Peled R, Yaari E, et al. The association between sociodemographic characteristics and adherence to breast and colorectal cancer screening: analysis of large sub populations. BMC Cancer 2011;11:376

81 Williams EM, Vessey MP. Randomised trial of two strategies offering women mobile screening for breast cancer. BMJ 1989;299:158-9.

82 Yarnall KS, Michener JL, Broadhead WE, et al. Increasing compliance with mammography recommendations: health assessment forms. J Fam Pract 1993;36:59-64.

83 Zackrisson S, Lindström M, Moghaddassi M, et al. Social predictors of non-attendance in an urban mammographic screening programme: a multilevel analysis. Scand J Public Health 2007;35:548-54.

84 Zidar MN, Larm P, Tillgren P, et al. Non-attendance of mammographic screening: the roles of age and municipality in a population-based Swedish sample. Int J Equity Health 2015;14:157.

85 Chan W, Yun L, Austin PC, et al. Impact of socio-economic status on breast cancer screening in women with diabetes: a population-based study. Diabet Med 2014;31:806-12.

86 Chochinov HM, Martens PJ, Prior HJ, et al. Does a diagnosis of schizophrenia reduce rates of mammography screening? A Manitoba population-based study. Schizophr Res 2009;113:95-100.

87 Vigod SN, Kurdyak PA, Stewart DE, et al. Depressive symptoms as a determinant of breast and cervical cancer screening in women: a population-based study in Ontario, Canada. Arch Womens Ment Health 2011;14:159-68.

88 Aro AR, de Koning HJ, Absetz P, et al. Psychosocial predictors of first attendance for organised mammography screening. $J$ Med Screen 1999;6:82-8.

89 Jensen LF, Pedersen AF, Andersen B, et al. Social support and nonparticipation in breast cancer screening: a Danish cohort study. $J$ Public Health 2016;38:335-42.

90 Lagerlund M, Sparén P, Thurfjell E, et al. Predictors of nonattendance in a population-based mammography screening programme; socio-demographic factors and aspects of health behaviour. Eur J Can Prev 2000;9:25-34.

91 Ulcickas Yood M, McCarthy BD, Lee NC, et al. Patterns and characteristics of repeat mammography among women 50 years and older. Cancer Epidemiol Biomarkers Prev 1999;8:595-9.

92 Dryden R, Williams B, McCowan C, et al. What do we know about who does and does not attend general health checks? findings from a narrative scoping review. BMC Public Health 2012;12:723.

93 Thomas VN, Saleem T, Abraham R. Barriers to effective uptake of cancer screening among black and minority ethnic groups. Int $\mathrm{J}$ Palliat Nurs 2005;11:562. 4-71.

94 Long $\mathrm{H}$, Brooks JM, Harvie M, et al. How do women experience a false-positive test result from breast screening? A systematic review and thematic synthesis of qualitative studies. $\mathrm{Br} \mathrm{J}$ Cancer 2019;121:351-8. 